\title{
Resonant Raman Spectra of CdTe/ZnTe Self Assembled Quantum Dots
}

\author{
N. Romčevića ${ }^{a *}$, M. Romčevića ${ }^{a}$, R. Kostićc $a$, D. Stojanovića ${ }^{a}$, B. Abolmasov ${ }^{b}$, \\ G. KARCZEWSKI ${ }^{c}$ AND R. GalazKA ${ }^{c}$ \\ ${ }^{a}$ Institute of Physics, Pregrevica 118, 11080 Belgrade, Serbia \\ ${ }^{b}$ Faculty of Mining and Geology, P.O. Box 162, 11000 Belgrade, Serbia \\ ${ }^{c}$ Institute of Physics, Polish Academy of Sciences \\ al. Lotników 32/46, 02-668 Warszawa, Poland
}

\begin{abstract}
In this paper we present Raman scattering and photoluminescence spectra measured on CdTe/ZnTe self assembled quantum dots. The photoluminescence spectrum has shown two main emission peaks, both connected with existing of quantum dots. One presents direct deexcitation to ground state and the other is optical phonon $\left(\omega=207.3 \mathrm{~cm}^{-1}\right)$-assisted deexcitation. The registered multiphonon emission process depends on temperature. At low temperature phonon spectra shows line at $450 \mathrm{~cm}^{-1}(\mathrm{TA}+2 \mathrm{LO}$ in $\mathrm{ZnTe})$ and $595 \mathrm{~cm}^{-1}(2 \mathrm{LO}+\mathrm{TO}(\Gamma)$ in $\mathrm{ZnTe})$.
\end{abstract}

PACS numbers: $63.22 .-\mathrm{m}, 78.30 . F s, 78.55 . E t$

\section{Introduction}

Great interest has been observed recently in studying the properties of layers of semiconductor materials with a mismatch between their lattice parameters. Favourable conditions for the formation of self-assembled quantum dots (QDs) are created in such layers [1]. The wide band-gap CdTe/ZnTe system is of current interest because of its potential applications in short-wavelength optoelectronic devices, but the large lattice mismatch (6.4\%) makes it very difficult to grow $\mathrm{CdTe} / \mathrm{ZnTe}$ structure of high quality [2].

Raman scattering has been widely used to study the phonons in low-dimensional semiconductor systems. For II-VI semiconductor, first order Raman scattering, multiphonon (MP) Raman scattering has received less attention [3]. For better understanding of optical and structural properties of $\mathrm{CdTe} / \mathrm{ZnTe}$ structures, in this paper we focused our attention to Raman and photoluminescence (PL) spectroscopy of $\mathrm{CdTe} / \mathrm{ZnTe}$ self organized quantum dots.

\section{Samples and characterization}

Investigated samples were grown by molecular beam epitaxy on GaAs substrate. CdTe buffer layer, $5 \mu \mathrm{m}$ thick, was deposited on the substrate. After a $0.8 \mu \mathrm{m}$ thick ZnTe layer, 12 monolayers (ML) of CdTe were deposited to form a random distribution of quantum

* corresponding author; e-mail: romcevi@phy.bg.ac.yu dots. High mismatch of lattice parameters of CdTe $(6.482 \AA)$ and ZnTe $(6.104 \AA)$ provokes gathering of CdTe molecules and forming of quantum dots. The dot layer was covered by $0.1 \mu \mathrm{m}$ ZnTe capping layer. Further details of the samples growth can be found in Ref. [1]. The Raman and photoluminescence spectra were excited by several lines of argon-laser line and measured using a Jobin Yvon model U-1000 monochromator, with a conventional photocounting system.

\section{Results and discussion}

The electronic band structure was characterized by a PL spectrum which showed two main emission peaks. Typical PL spectra, recorded at $200 \mathrm{~K}$, are presented in Fig. 1. The peaks positions are $2.307 \mathrm{eV}\left(E_{1}\right)$ and $2.11 \mathrm{eV}\left(E_{2}\right)$ at $T=200 \mathrm{~K}$, and $2.337 \mathrm{eV}\left(E_{1}\right)$ and $2.13 \mathrm{eV}\left(E_{2}\right)$ at $T=20 \mathrm{~K}$. Their energies decrease with temperature increase, as presented in Fig. 2. The first peak, $E_{1}$, is related to optical phonon $\left(\omega=207.3 \mathrm{~cm}^{-1}\right)$ assisted emission. MP resonant processes are registered at temperature below $200 \mathrm{~K}$. This process is dominant for QDs with high emission energy, i.e. presumably with smaller size. The second one, $E_{2}$, is related to direct excited state-ground state transition. This process is important for all QDs.

Calculation of electronic transitions is done in common effective-mass approximation [4]. Parameters, effective masses and conduction and valence offsets are transferred from literature. In our case we use approximation that supports the assumption which leads from PL spectra. We suppose that electrons and holes from QD are 


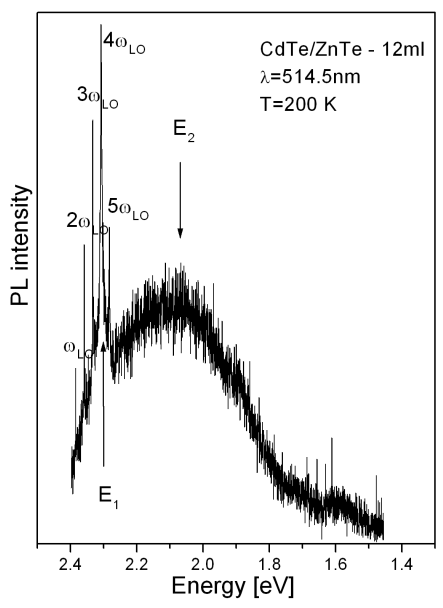

Fig. 1. Typical PL spectra of sample with $12 \mathrm{ML}$ of CdTe in the finishing layer; PL spectra are excited by $\lambda=514.5 \mathrm{~nm}$ Ar-laser line and recorded at $200 \mathrm{~K}$.

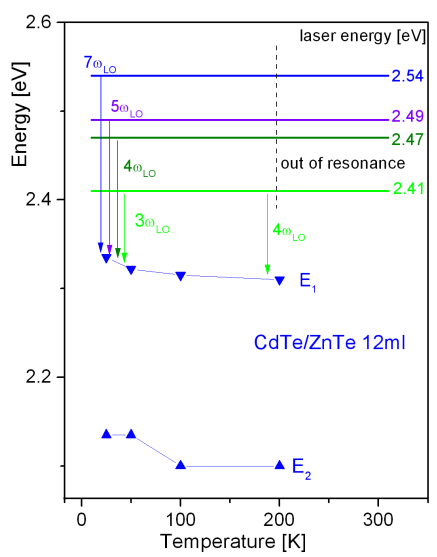

Fig. 2. Schematic presentation of multiphonon resonance and temperature dependences of PL emission peaks.

in step-like energy well. CdTe is in the core and the concentration of CdTe in the surrounding $\mathrm{Cd}_{x} \mathrm{Zn}_{1-x} \mathrm{Te}$ gradually decreases to $10 \%$ ( $x=0.1$ ), step by step. For measured $E_{2}$ the dimension of the well core, related with dimension of $\mathrm{QD}$, is $1.55 \mathrm{~nm}$ and the external diameter of the energy well is $6.48 \mathrm{~nm}$. Broad $E_{2}$ band in this sample suggests the great dispersion in dimensions of QDs, i.e. the great variations in relief of CdTe layer.

In principle, registered MP resonant process (see Fig. 3) can be described by the following equation: $E_{\text {in }}-K \hbar \omega=E ; E_{\text {in }}$ and $E$ are the incident photon energy and electron transition energy, respectively. $\hbar \omega$ is the phonon energy and $K$ is the MP order. MP emission is not registered at $T=300 \mathrm{~K}$. MP emission processes are registered at temperature below $T=200 \mathrm{~K}$ (for $2.41 \mathrm{eV}$ laser energy, $K=4)$. The equation means that when the energy of the scattered photon approached the energy $E$, the Raman line becomes strongly enhanced. At $T=20 \mathrm{~K}$ resonance order depends on excitation energy

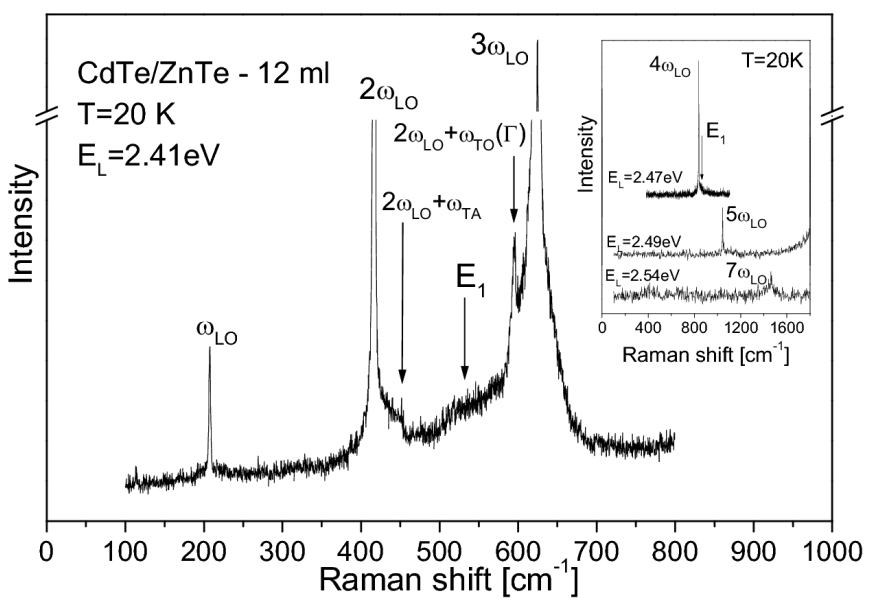

Fig. 3. Raman spectra of $\mathrm{CdTe} / \mathrm{ZnTe}$ self assembled $\mathrm{QD}$ at $T=20 \mathrm{~K}$. Inset: PL spectra excited by different excitation energies at $T=20 \mathrm{~K}$.

and its values are $3,4,5$ and 7 for laser lines $2.41 \mathrm{eV}$, $2.47 \mathrm{eV}, 2.49 \mathrm{eV}$ and $2.54 \mathrm{eV}$, respectively (see inset in Fig. 3), which is schematically presented in Fig. 2.

Phonon spectra depend on temperature. More complicate spectra are registered at $T=20 \mathrm{~K}$, Fig. 3 . Beside the phonon at $207.3 \mathrm{~cm}^{-1}$, its harmonics, which participate in MP processes, lines at $450 \mathrm{~cm}^{-1}$ and $595 \mathrm{~cm}^{-1}$ can easily be observed. These modes can be assigned in this way: we attribute the peak $207.3 \mathrm{~cm}^{-1}$ to confine longitudinal-optical (LO) phonons from bulk $\mathrm{ZnTe}$ $\left(209 \mathrm{~cm}^{-1}\right)$ [5]. We identified the peaks at $450 \mathrm{~cm}^{-1}$ and $595 \mathrm{~cm}^{-1}$ as ZnTe $(\mathrm{TA}+2 \mathrm{LO})$ modes and $(2 \mathrm{LO}+\mathrm{TO}(\Gamma))$ modes, respectively.

\section{Conclusion}

In this paper we investigated structural and optical properties of $\mathrm{CdTe} / \mathrm{ZnTe}$ self-assembled quantum dots growth by molecular beam epitaxy. Photoluminescence spectra consist of two main emission peaks: direct excited state-ground state transition $\left(E_{2}\right)$ and optical phonon assisted emission $\left(E_{1}\right)$. The registered multiphonon emission processes depend on temperature. When the energy of the scattered photon approaches the energy $E_{1}$, the corresponding Raman line becomes strongly enhanced. At low temperature phonon spectra shows $\omega_{\text {LO }}$ line at $207.3 \mathrm{~cm}^{-1}$, its higher order harmonics and multiphonon modes at $450 \mathrm{~cm}^{-1}(\mathrm{TA}+2 \mathrm{LO}$ in $\mathrm{ZnTe})$ and $595 \mathrm{~cm}^{-1}$ $(2 \mathrm{LO}+\mathrm{TO}(\Gamma))$.

\section{Acknowledgments}

This work was supported under the Agreement of Scientific Collaboration between Polish Academy of Sciences and Serbian Academy of Sciences and Arts. The work in Serbia was supported by Serbian Ministry of Science and Technologial Development (projects No. 141028). 


\section{References}

[1] S. Mackowski, Thin Solid Films 412, 96 (2002).

[2] S. Mackowski, G. Karczewski, T. Wojtowicz, J. Kossut, S. Kret, P. Dluzewski, Appl. Phys. Lett. 78, 3884 (2001).

[3] A.A. Arora, M. Rajalakshmi, T.R. Ravindran, V. Sivasubramanian, J. Raman Spectrosc. 38, 604 (2007).
[4] M. Tkach, V. Holovatsky, O. Voitsekhivska, M. Mikhalyova, Phys. Status Solidi B 203, 373 (1997).

[5] S.L. Zhang, Y.T. Hou, M.Y. Shen, J. Li, S.X. Yuan, Phys. Rev. B 47, 12937 (1993). 